\title{
Development of drug resistance in Trypanosoma brucei rhodesiense and Trypanosoma brucei gambiense. Treatment of human African trypanosomiasis with natural products (Review)
}

\author{
STEFANIE GEHRIG $^{1}$ and THOMAS EFFERTH ${ }^{2}$ \\ ${ }^{1}$ Institute of Pharmacy and Molecular Biotechnology, University of Heidelberg, Im Neuenheimer Feld 364, ${ }^{2}$ German \\ Cancer Research Center, Pharmaceutical Biology (C015), Im Neuenheimer Feld 280, 69120 Heidelberg, Germany
}

Received April 10, 2008; Accepted June 12, 2008

DOI: 10.3892/ijmm_00000037

\begin{abstract}
Human African trypanosomiasis is an infectious disease which has resulted in the deaths of thousands of people in Sub-Saharan Africa. Two subspecies of the protozoan parasite Trypanosoma brucei are the causative agents of the infection, whereby $T . b$. gambiense leads to chronic development of the disease and $T . b$. rhodesiense establishes an acute form, which is fatal within months or even weeks. Current chemotherapy treatment is complex, since special drugs have to be used for the different development stages of the disease, as well as for the parasite concerned. Melarsoprol is the only approved drug for effectively treating both subspecies of human African trypanosomiasis in its advanced stage, however, the drug's potency is constrained due to an unacceptable side effect: encephalopathy, which develops in one out of every 20 patients who are treated with the drug. In addition to the deleterious treatment with melarsoprol, the number of drugresistant strains of T. brucei supp. increases. Mechanisms of drug resistance have been elucidated and involve decreased drug import through the loss of the purine transporter P2 as well as enhanced drug export, mediated by a multidrug resistance-associated protein called TbMRPA. Thereby, the medical treatment with the available chemotherapeutics becomes exceedingly difficult. A promising strategy for research into new drugs and moreover, to overcome drug resistance, are compounds derived from natural sources. This study provides an overview of the recently discovered small molecules with trypanocidal activity against $T . b$. gambiense and $T . b$. rhodesiense. In addition, former promising compounds are touched upon.
\end{abstract}

Correspondence to: Dr Thomas Efferth, German Cancer Research Center, Im Neuenheimer Feld 280, 69120 Heidelberg, Germany E-mail: t.efferth@dkfz.de

Key words: $\alpha$-difluorornithine, chemotherapy, drug resistance, human African trypanosomiasis, melarsoprol, natural products, pentamidine, pharmacognosy, suramin, trypanocidal activity, Trypanosoma brucei gambiense, Typanosoma brucei rhodesiense

\section{Contents}

1. Introduction

2. Disease and clinical manifestation

3. Standard chemotherapy

4. Development of resistance

5. Screening of natural products

6. Conclusion

\section{Introduction}

Human African typanosomiasis is a vector-born parasitic disease demonstrating a major public health problem for people in the Sub-Saharan region. The transmission of the disease is heightened due to lack of hygiene and close relationship between humans and domestic mammals. Furthermore, endemics develop in rural areas where medical facilities needed for an effective treatment are missing (1). Resistance is a broad problem in the fight against this infectious disease. Except for the limited drugs used currently in chemotherapy, no other drugs are available, if strains were to develop resistance.

The search for new drugs is difficult and drug development generally takes years. The easiest strategy is derivatisation of approved drugs, to achieve improved pharmacokinetics and changes in the anti-protozoal activity. Nevertheless, the mode of action stays the same, and resistant strains are not highly sensitive to derivatives and frequently develop resistance to structurally related substances as well. Another problem is that standard chemotherapeutics often exhibit unconfirmed risks to patients. Due to serious side effects maximal dosages, needed for the complete eradication of the parasite, and thereby, avoiding development of resistance, are deleterious (2). This shows a strong need for research into novel drugs for the treatment of human African trypanosomiasis, to develop safe, effective drugs, and to overcome drug resistance.

Active substances can chemically be synthesized or, alternatively, can also be identified in natural sources with high diversity of chemical structures. The most common of which is the use of plant-derived compounds in medical application, but even fungi, bacteria, and animals can be a source for chemical substances with potency for treatment of diseases (3). 
Of special interest are compounds, which are not generally cytotoxic, but show trypanocidal specificity. By serving as novel lead compounds, their structures can be optimized by semi-synthetic or fully synthetic derivatization (4). In the fields of pharmacology and pharmacy, natural products have always played an important role in combating diseases. Famous examples are the glycosides digitoxin, isolated from Digitalis purpurea and digoxin, derived from $D$. lanata, which are applied against cardiac diseases. Other examples are demonstrated by the alkaloids vincristine and vinblastine, purified from the plant Vinca rosea (Catharanthus roseus) and paclitaxel, isolated from Taxus brevifolia, which are naturally derived anticancer drugs (1). These successes demonstrate that natural products are a potent source for the development of drugs against a variety of diseases and in particular, to give an impact on the treatment of human African trypanosomiasis by drugs that follow different mechanisms of action, thereby, increasing their efficiency and avoiding development of drug resistance. Other strategies to limit the number of infections are either to address the parasite in its vector or to combat the vector itself (5).

\section{Disease and clinical manifestation}

Human African trypanosomiasis, also known as sleeping sickness, has been resurgent in Sub-Saharan Africa since the late 1990s (5), and today 50 million people are at risk of becoming infected with the disease (6). Although, progress in disease control has been made, the World Health Organization (WHO) estimated in 2005 that the number of infections lies between 50,000-70,000 (7). Human African trypanosomiasis is caused by two morphologically indistinguishable subspecies of the hemoflagellated protozoan parasite Trypanosoma brucei, which are transmitted to the human host each by a specific species of the genus Glossina, known as tsetse fly. Both vectors inhabit distinct areas of Africa, leading to an epidemiological division of the causative agents of the disease. In West Africa, T. b. gambiense is transmitted primary to humans by G. palpalis, whereas in East Africa G. morsitans play a role as a vector for incidental transmission of T. b. rhodesiense to humans, while generally infecting domestic mammals (6).

According to the appearance of trypanosomes in the vector and the mammalian host, the life cycle of Trypanosoma brucei is digenetic. Procyclic forms of trypanosomes exist in the tsetse flies' mid gut, where they develop into epimastigotes and leave the mid gut to transform into metacyclic infective trypomastigotes in the salivary glands of the fly. The mammalian host gets involved in the life cycle during a blood meal of the fly. Thereby, metacyclic trypomastigotes are inoculated into the skin and infest the blood system. In the host, they transform into bloodstream trypomastigotes, which divide by binary fission in the blood, lymph and extra cellular spaces. Characteristic for the African trypanosomiasis is that no intracellular form exists. The parasites re-enter the tsetse fly, when an infected mammal is bitten again and redevelop into procyclic trypomastigotes (8). For the infected human the disease develops from a first hemolymphatic stage with mild symptoms, including fever, headaches, joint pains and itching, into a second stage, where the parasites cross the blood brain barrier and establish a cerebral infection.
At the second stage, being more adverse, the patient suffers from torture of the central nerve system. Severe headaches, loss of nocturnal sleep, mental disorientation, tremors of tongue, hands and feet, and any neurological psychiatric manifestation are the outcome. Finally, patients fall into coma and die of opportunistic infections (6). The timed development of symptoms and the life expectancy strongly depend on the $T$. brucei subspecies with which the human is infected. A chronic disease is caused by $T . b$. gambiense, which is mostly asymptomatic for months or even years. When symptoms appear, the parasites have already infected the central nervous system. In contrast, an acute infection is caused by $T . b$. rhodesiense within weeks or months. In this case, the disease rapidly affects the central nervous system and is fatal for untreated patients (7).

In order to approach the defense against the protozoan parasite, differences in biochemical mechanisms between parasite and host are promising drug targets without expected cytotoxicity towards the human host (9). A particularity of Trypanosoma is constituted by its single mitochondrion, which possesses a high number of circular DNA, organized to an interlocked and super-coiled network, called kinetoplast DNA (kDNA). As a result, Trypanosoma is classified as a Kineplastida. The kDNA renders the mitochondrial replication as a high energetic process, and while divergent to the human host, it serves as a potential drug target (10).

To manage the carbohydrate metabolism, Trypanosoma has developed alternative pathways, including the glycosome. This is a special organelle in which part of the glycolysis is compartmentalized (11). Another specific characteristic is demonstrated by trypanothione and trypanothione reductase, both essential for the parasite's defense against oxidative stress (12). Trypanothione is built by a covalent complex of two glutathiones linked by the polyamine spermidine (13). The polyamine synthesis, maintained by the enzymes $\mathrm{S}$-adenosylmethionine decarboxylase (SAMDC) and ornithine decarboxylase (ODC), is therefore also a potential drug target. Alteration leads to an impaired generation of trypanothione, whereby the parasite becomes susceptible to oxidative stress. $(14,15)$. In addition, trypanosomes lack de novo purine synthesis as well as synthesis of cholesterol and fatty acids, making the parasite obligated to supply of these molecules from the host $(16,17)$.

Given that the parasites stay extracellular, the host's immune system recognizes an infection with T. brucei supp., initiating a humoral immune response (3). Nevertheless, eradication is not achieved, since the parasite evades the immune system mainly by switching of variant surface glycoproteins (VSG) each generation (15). Thereby, the generation of antibodies no longer recognizes the parasites' surface antigens, enabling the parasite always to be one step in front of the hosts' immune defense mechanisms, therefore, all efforts to develop a vaccine against trypanosomiasis have not been effective (3).

\section{Standard chemotherapy}

The treatment of human African trypanosomiasis is challenging due to the fact that drugs have to be efficient against the two subspecies of $T$. bruce $i$ and the two developmental stages of 
the disease. To fulfill these demands, chemotherapy has to be co-ordinated to each case (18). Pentamidin, a water soluble aromatic diamidine with amidine groups at either end, is applied in the early stage of the disease (15), but its efficiency is restricted to T. b. gambiense (19). Pentamidin enters the parasite via the purine transporter $\mathrm{P} 2$, a protein primarily responsible for the transport of adenosine and adenine (20). Due to the transported molecules and drugs, the recognition motif is likely to be constituted by amidine nitrogens (21).

Pentamidine possesses trypanocidal activity by binding to a minor groove of kDNA in the mitochondrion, thereby, inducing changes in DNA topology and inhibiting topoisomerases $(22,23)$. As a result, the replication of the mitochondrion is impaired and DNA cleavage occurs. Moreover, pentamidin has been identified as inhibitor of the S-adenosylmethionine decarboxylase (24), resulting in inhibition of the polyamine synthesis and in turn lack of trypanothione. Suramin, a sulfonated naphthylamine, is the second early stage drug applied in infections with $T . b$. rhodesiense (25). Exhibiting binding affinities to many plasma proteins, including low density lipoprotein (LDL) (25), suramin is taken up via receptor-mediated LDL endocytosis. It has been shown to inhibit various glycolytic enzymes, as well as receptor-mediated LDL uptake, among other activities $(26,27)$.

Its trypanocidal action is probably constituted by inhibition of LDL uptake, prohibiting the parasite's supply of cholesterol and phospholipids. For the treatment of late stage human African trypanosomiasis melarsoprol, a melaminophenyl arsenical, is the drug of first choice by providing efficiency against $T . b$. gambiense and T. b. rhodesiense (28). After its uptake into the trypanosome via P2 (15) melarsoprol is degraded to melarsen oxide, a metabolite which has been shown to be highly toxic in vivo and causing in rapid lysis of the parasites (29). Furthermore, melarsoprol interacts with trypanothione forming a stable complex, called MelT, which in turn is an inhibitor of the trypanothione reductase (30,31).

Inhibition of trypanothione reductase and decrease of trypanothione level by formation of adducts act two-fold to impair the parasite's defence against oxidative stress. There is no doubt that the application of melarsoprol is crucial to combat late stage infections, but the application of this drug is hampered by its adverse side effects (28). Most prominently, encephalopathy causes mortality in five percent of treated patients (3). A newer late stage drug, $\alpha$-difluoromethylornithine (DMFO, eflornithine) (32), is safer, but only possesses efficiency against $T$. b. gambiense (33). It enters the parasite via passive diffusion (34) and acts as an irreversible inhibitor of the ornithine decarboxylase (ODC) $(35,36)$. Thereby, the polyamine synthesis is inhibited, resulting in a decreased level of redox regulator trypanothione (19).

It has been elucidated that the lack of efficiency against $T$. $b$. rhodesiense is caused by a faster turnover rate of its ODC-enzymes (34). As a result, inhibited ODCs are replaced faster in $T . b$. rhodesiense. Additionally, substrates in the pathway of polyamine synthesis are accumulated, and in particular, the S-adenosylmethionine level increases, which might disrupt normal methylation patterns of proteins and other cell components (37).

\section{Development of resistance}

The development of drug resistance is caused by different mechanisms. Altered metabolic pathways prevent the drug from reaching its target. Alternative pathways facilitate the parasites' independence on inhibited targets or detoxification of the drug. In addition, intracellular accumulation of the drug can be inhibited by decreased drug import or increased drug export (38).

Due to the extensive use of drugs and their structural similarities, multiple drug resistance has developed (3). Resistance in $T . b$. rhodesiense and $T . b$. gambiense is frequently mediated by reduced net drug uptake. Reduced drug import is mediated primarily via loss of $\mathrm{P} 2$, also named TbAT1 (for Trypanosoma brucei adenosine transporter 1) (15). Additionally, silent and coding point mutations in the TbAT1 gene lead to changes in substrate specificity (39).

Surprisingly, loss of P2 induces only a small increase of resistance towards melarsoprol and pentamidine (2-3-fold) (18). This might be due to a P2-independent uptake of melarsoprol and pentamidine by additional transporters. One transporter identified is HAPT1 (high-affinity pentamidine transporter), which mediates supplemental import of melarsoprol. Therefore, high-level melarsoprol resistance might be caused by loss of both transporters, P2 and HAPT1 (18).

Another drug resistance mechanism for melarsoprol represents the overexpression of the efflux pump TbMRPA (10-fold) $(15,40)$. TbMRPA is one of three ATP-binding cassette transporters present in Trypanosoma brucei (41). It belongs to the sub-family of multidrug resistance-associated proteins (MRPs), characterized by the need of ATP and thiols to transport molecules against a concentration gradient (42). TbMRPA functions as a detoxification efflux pump localized in the plasma membrane of the parasite and mediates the efflux of MelT $(15,40)$.

Other resistance mechanisms, unrelated to drug transport, have also been observed emphasizing the multifactorial nature of drug resistance (15). As a result, emergence of drug resistance hinders efforts to control the disease (18). Increase in treatment failures with melarsoprol, and more importantly, its unacceptable side effect encephalopathy, shows the urgent need for new trypanocidal agents (3). Due to the fact that DMFO is not effective against $T . b$. rhodesiense, there is no safe and efficient drug that crosses the blood-brain barrier to treat the late stage human African trypanosomiasis. In addition, early stage chemotherapeutics have to be administered parenterally, which makes the treatment impossible in areas, where no medical facilities exist (43).

\section{Screening of natural products}

The potential of natural products in the treatment of diseases can be seen in traditional medicines. Since treatments with medicinal plants were developed over thousands of years and are still practised today, it might be promising to search for plants traditionally used to treat sleeping sickness and to investigate their bioactive chemical constituents (1). To permit meaningful investigations on a molecular level, it is important to focus strictly on isolated compounds from plant raw extracts (44). 
A previous review of Hoet and co-workers presents a wide variety of compounds isolated from natural sources with trypanocidal activity reported in publications from mid-1980 to June 2003 (45). Therefore, the present overview focuses on a summary of the latest results in the field and concomitantly a comprehensive overview is provided by displaying the most promising compounds again. Furthermore, the present overview is restricted to compounds from natural products exhibiting activity against $T . b$. gambiense and $T . b$. rhodesiense. Beside the determination of the trypanocidal effect itself, most studies in the literature also describe the cytotoxicity of natural products towards a mammalian cell line.

Taking both measures into account, the selectivity index (SI) can be calculated by dividing the $\mathrm{IC}_{50}$ value of a compound for a mammalian cell line through the $\mathrm{IC}_{50}$ for its trypanocidal action. The SI indicates, whether a compound is generally cytotoxic (low SI) or specifically affects the parasite (high SI). Compounds with high SI values are suitable for in vivo studies. The reader should keep in mind that the data in the literature were generated in different experimental settings. Therefore, the values are not fully comparable.

Where possible, we indicate the activity of a reference drug, melarsoprol, to allow the reader at least some comparability between different investigations. To constrain the huge number of compounds reported in the literature, only those which possess trypanocidal activity in concentrations $<80 \mu \mathrm{M}$ were considered in the present review. The trypanocidal substances are organised according to their chemical structure (Figs. 1-4).

Alkaloids (Fig. 1): Ancistrolikokine D [1], a naphthylisoquinoline alkaloid, was isolated from Ancistrocladus likoko (Ancistrocladaceae), a tropical liana indigenous to the rainforests of Central Africa. The $\mathrm{IC}_{50}$ value was $2.71 \mu \mathrm{g} / \mathrm{ml}$ $(6.9 \mu \mathrm{M})$ on bloodstream forms of $T . b$. rhodesiense, and a SI value of 13.5 was obtained when compared to mammalian L6 cells (rat skeletal myoblasts). For the reference drug, melarsoprol, an $\mathrm{IC}_{50}$ value of $0.0026 \mu \mathrm{g} / \mathrm{ml}(0.007 \mu \mathrm{M})$ was obtained (46).

Several naphthylisoquinoline alkaloids, ancistrocladidine [2], ancistrotectorine [3], ancistrotanzanine C [4], O-methylancistrocladinine [5] and $O, N$-dimethylancistrocladine [6], were isolated from Ancistrocladus tarzanienses. The $\mathrm{IC}_{50}$ values were $2.0,4.3,1.3,5.2$ and $5.4 \mu \mathrm{g} / \mathrm{ml}(4.7,9.9,3.2$, 12.4 and $12.4 \mu \mathrm{M})$, respectively, on bloodstream forms of T. b. rhodesiense, and SI values were 14.2, 4.6, 31.3, >17, and 7.9 when compared to L6 cells. For the reference drug, melarsoprol, an $\mathrm{IC}_{50}$ value of $0.0031 \mu \mathrm{g} / \mathrm{ml}(0.008 \mu \mathrm{M})$ was obtained (47).

In addition to these studies, highly structurally related naphthylisoquinoline alkaloids were isolated from another species of Ancistrocladus. Ent-dioncophylleine A [7], 5'-Odemethyl-ent-dioncophylleine A [8] and dioncophylleine D [9], derived from A. benomensis, exhibited $\mathrm{IC}_{50}$ values of 5.6, 1.4 and $4.7 \mu \mathrm{g} / \mathrm{ml}(15.0,3.9$ and $12.6 \mu \mathrm{M})$ on bloodstream forms of $T . b$. rhodesiense. In the applied concentrations 5'-O-demethyl-ent-dioncophylleine possessed cytotoxicity to L6 cells with a SI value of 14.6. For the reference drug, melarsoprol, an $\mathrm{IC}_{50}$ value of $0.00095 \mu \mathrm{g} / \mathrm{ml}(0.002 \mu \mathrm{M})$ was obtained (48).
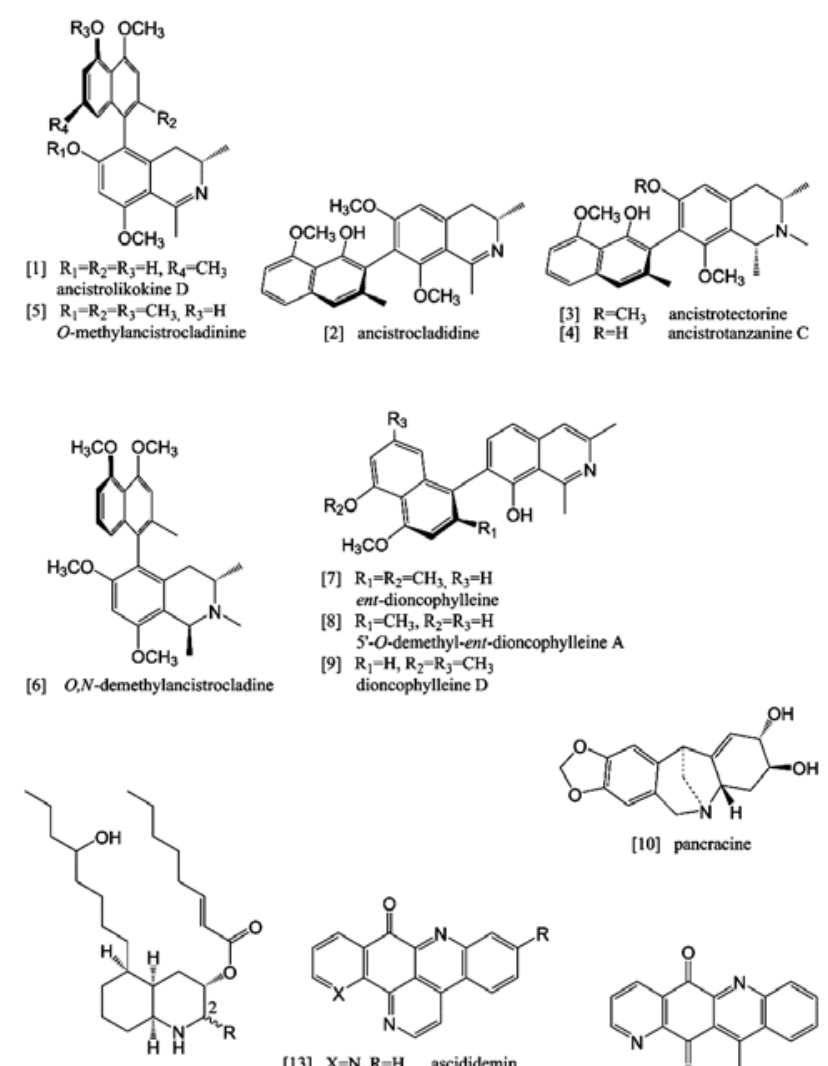

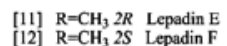

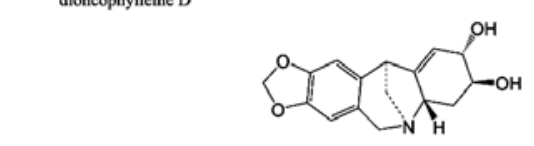

[10] pancracine

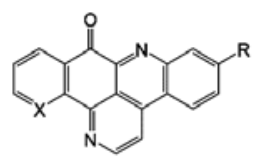

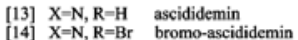

[15] $\mathrm{X}=\mathrm{CH}, \mathrm{R}=\mathrm{H}$ synthetic derivative $\mathrm{A}$

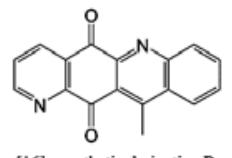

Figure 1. Chemical structures of alkaloids with trypanocidal activity.

Pancracine [10], an isoquinone alkaloid, was isolated from the bulb of Narcissus augustifolius transcarpathius (Amaryllidaceae). The $\mathrm{IC}_{50}$ value was $0.7 \mu \mathrm{g} / \mathrm{ml}(2.4 \mu \mathrm{M})$ on bloodstream forms of $T$. $b$. rhodesiense. At concentrations $\leq 300 \mu \mathrm{M}$, no cytotoxicity was observed when compared to L6 cells. For the reference drug, melarsoprol, an $\mathrm{IC}_{50}$ value of $0.0014 \mu \mathrm{g} / \mathrm{ml}(0.004 \mu \mathrm{M})$ was obtained (49).

Lepadin E [11] and lepadin F [12], both decahydroquinolines derivates, were isolated from a novel species of the tunicate Didemnum (Didemnidae). The $\mathrm{IC}_{50}$ values for the two diastereoisomers were 0.38 and $0.23 \mu \mathrm{g} / \mathrm{ml}(0.9$ and $0.5 \mu \mathrm{M}$ ), respectively, on bloodstream forms of $T . b$. rhodesiense. The compounds showed moderate selectivity towards the parasite with SI values of 43 and 80 when compared to L6 cells. For the reference drug, melarsoprol, an $\mathrm{IC}_{50}$ value of $0.0026 \mu \mathrm{g} / \mathrm{ml}(0.007 \mu \mathrm{M})$ was observed (50).

Ascididemin [13] and 2-bromoascididemin [14], two pyridoacridone alkaloids derived from a marine organism, possessed trypanocidal activity in vitro. Ascididemin is known to exhibit anti-tumor activity by DNA intercalation. The $\mathrm{IC}_{50}$ values were 4 and $1.76 \mu \mathrm{g} / \mathrm{ml}(14$ and $5 \mu \mathrm{M})$, respectively, on bloodstream forms of $T$. b. rhodesiense, and SI values of 0.1 and 0.4 were observed when compared to L6 cells. For the reference drug melarsoprol an $\mathrm{IC}_{50}$ value of $0.0012 \mu \mathrm{g} / \mathrm{ml}$ $(0.003 \mu \mathrm{M})$ was obtained. The presence of a benzonaphthyridone substructure seems to be important for the trypanocidal activity. It has been suggested that the compounds intercalate into the DNA and induce oxidative damage. Nevertheless, the potential of the substances is limited due to 

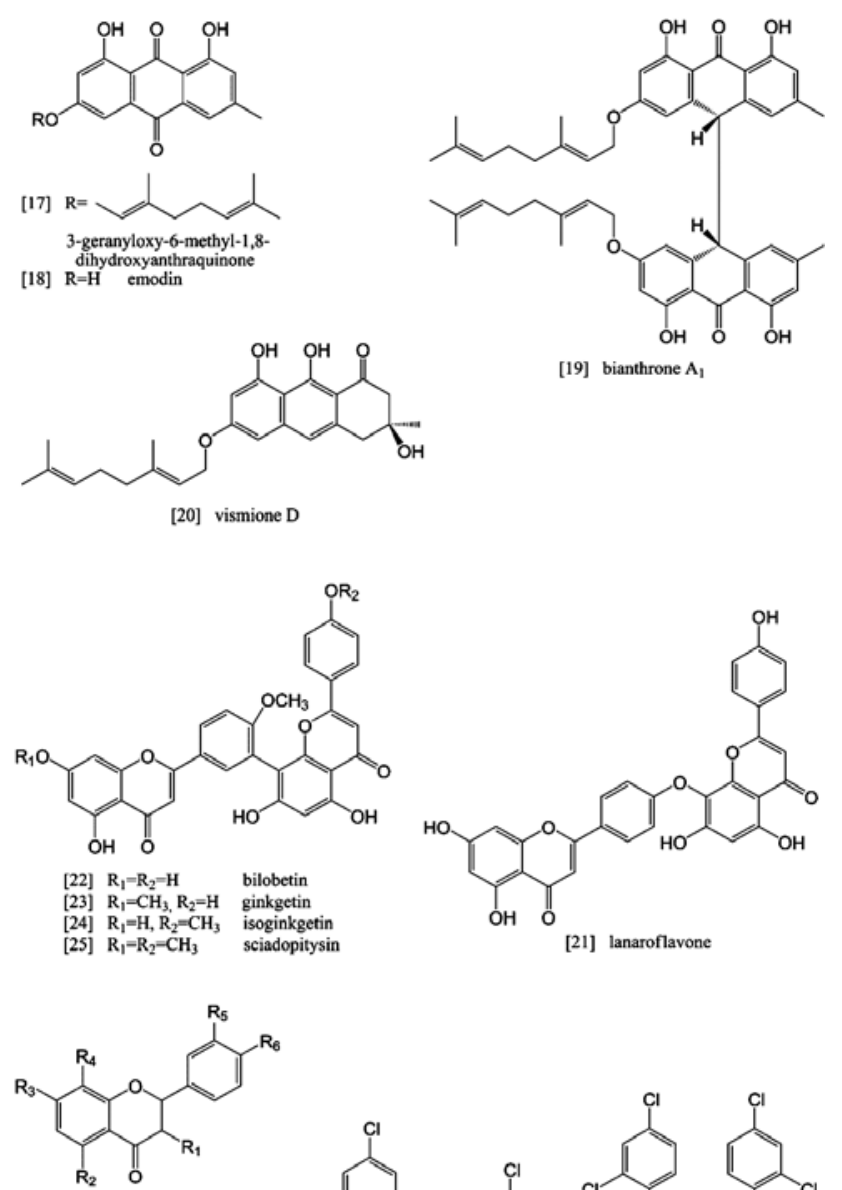

[28] $\mathrm{R}_{1}=\mathrm{R}_{2}=\mathrm{R}_{5}=\mathrm{R}_{6}=\mathrm{H}, \mathrm{R}_{3}=\mathrm{R}_{4}=\mathrm{OH}$ [29] $\mathrm{R}_{1}=\mathrm{OH}, \mathrm{R}_{2}=\mathrm{R}_{3}-\mathrm{R}_{4}=\mathrm{R}_{5}-\mathrm{R}_{6}=\mathrm{H}$ [30] $\mathrm{R}_{1}=\mathrm{R}_{2}=\mathrm{R}_{5}=\mathrm{R}_{6}=\mathrm{OH}, \mathrm{R}_{3}=\mathrm{R}_{4}=\mathrm{H}$

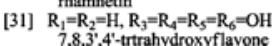

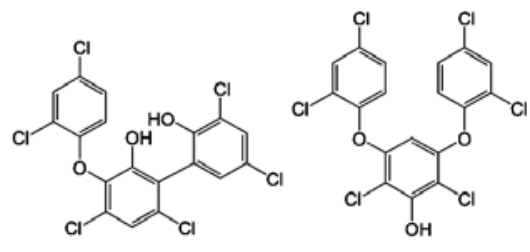

[26] ambigol A

[27] ambigol C

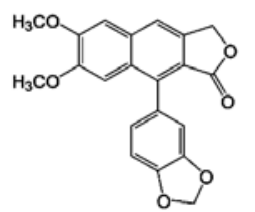

[32] justicidin B

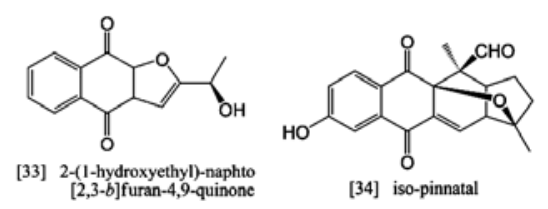

Figure 2. Chemical structures of phenolics with trypanocidal activity.

their high cytotoxicity. By synthetic derivatization related compounds were obtained, which possessed significantly higher specificity towards the parasite. For two compounds $[15,16]$ the $\mathrm{IC}_{50}$ values were 0.002 and $0.005 \mu \mathrm{g} / \mathrm{ml}(0.007$ and $0.018 \mu \mathrm{M})$, respectively, on bloodstream forms of $T . b$. rhodesiense, and SI values 950 and 316 were observed when compared to L6 cells (51).

Phenolics (Fig. 2): 3-geranyloxy-6-methyl-1,8-dihydroxyanthraquinone [17], emodin [18] and bianthrone A1 [19], all anthraquinones, as well as vismione D [20], an tetrahydroanthracene derivative, were isolated from the stem bark of Vismia orientalis (Guttiferae or Clusiaceae), a plant used in traditional medicine in Tanzania. Vismione D exhibited moderate activity with an $\mathrm{IC}_{50}$ value of $9 \mu \mathrm{g} / \mathrm{ml}(22 \mu \mathrm{M})$. However, a rather low SI value of 2.2 was observed when compared to L6 cells. Less trypanocidal activity was displayed by the three anthaquinone derivatives with $\mathrm{IC}_{50}$ values of $14.4,18.1$ and $53.5 \mu \mathrm{g} / \mathrm{ml}(35.3,66.8$ and $68.4 \mu \mathrm{M})$, respectively. Only emodin possessed cytotoxicity on L6 cells with an SI value of 1.1. Interestingly, bianthrone A1 which is a dimer of 3-geranyloxy-6-methyl-1,8-dihydroxyanthraquinone possessed only half of the trypanocidal activity of its monomeric molecule. For the reference drug, melarsoprol, an $\mathrm{IC}_{50}$ value of $0.0015 \mu \mathrm{g} / \mathrm{ml}(0.004 \mu \mathrm{M})$ was obtained (52).

Lanaroflavone [21], a biflavonoid, was isolated from Campnosperma panamense (Anacardiaceae) collected in Southern Columbia. Other biflavonoids, bilobetin [22], ginkgetin [23], isoginkgetin [24] and sciadopitysin [25], were derived from Ginkgo biloba (Ginkgoaceae). The $\mathrm{IC}_{50}$ values were $27,8.9,7.0,3.5$ and $7.0 \mu \mathrm{M}$, respectively, on bloodstream forms of $T . b$. rhodesiense, and SI values were 2.6, 2.9, 1.3, 3.1 and 9.7 when compared to L6 cells. Therefore, isoginkgetin possessed the highest trypanocidal activity, but without adequate specificity towards the parasite. For the reference drug, melarsoprol, an $\mathrm{IC}_{50}$ value of $0.005 \mu \mathrm{M}$ was obtained (53).

Ambigol A [26] and ambigol C [27], two chlorinated aromatic compounds, were isolated from the terrestrial cyanobacterium Fischerella ambigua. The minimal inhibitory concentrations (MIC) were 33 and $11 \mu \mathrm{g} / \mathrm{ml}(68 \mu \mathrm{M}$ and $22.7 \mu \mathrm{M}$ ), respectively, on bloodstream forms of $T . b$. rhodesiense. Whereas ambigol A showed no selectivity toward the parasite with an SI value of 1 when compared to L6 cells, the SI value for ambigol C was 9.1. No reference drug was used (54).

Tasdemir and coworkers screened a large number of flavonoids and analogues for their trypanocidal activity in vitro. The most potent compound was the natural product 7,8 dihydroxyflavone [28], followed by 3-hydroxyflavone [29], rhamnetin [30] and 7,8,3',4'-tetrahydroxyflavone [31] with $\mathrm{IC}_{50}$ values of $0.068,0.5,0.5$ and $0.5 \mu \mathrm{g} / \mathrm{ml}(0.27,2.0,1.7$, and $1.7 \mu \mathrm{M}$ ), respectively, on bloodstream forms of $T . b$. rhodesiense and SI values of 116.2, 28.8, >180, and 43.2 when compared to L6 cells. Additionally, the in vivo efficiency of several compounds was tested in a study with NMRI mice infected with T. b. brucei STIB 795. Application of 7,8dihydroxyflavone i.p. $50 \mathrm{mg} / \mathrm{kg}$ twice per day for 4 days could not eradicate the parasites, but prolong the survival for six days compared to the untreated control group. Treatment under same conditions with 3-hydroxyflavone had no effects. The positive control, melarsoprol, administered i.p. $2 \mathrm{mg} / \mathrm{kg}$ for 4 days cured infected mice (44).

Justicidin B [32], an arylnaphthalide lignan, was isolated as the main active principle of Phyllanthus piscatorum (Euphorbiaceae). The raw extract of the plant is used for topical treatment of Candida albicans infections of the vagina. The $\mathrm{IC}_{50}$ value was $0.2 \mu \mathrm{g} / \mathrm{ml}(0.55 \mu \mathrm{M})$ on bloodstream forms of $T . b$. rhodesiense with an SI value of 16.5 when compared to L6 cells. However, a high cytotoxicity demonstrated by an SI value of 1 was observed when compared to HeLa cells. For the reference drug, melarsoprol, an $\mathrm{IC}_{50}$ value of $0,003 \mu \mathrm{g}$ / $\mathrm{ml}(0.0008 \mu \mathrm{M})$ was obtained (55).

2-(1-hydroxyethyl)-naphato[2,3-b]furan-4,9-quinone [33], a furanonaphtoquinone and iso-pinnatal [34], a naphthoquinone, were isolated from Kigelia pinnata (Bignoniaceae). In Africa $K$. pinnata is a medial plant for the treatment of 


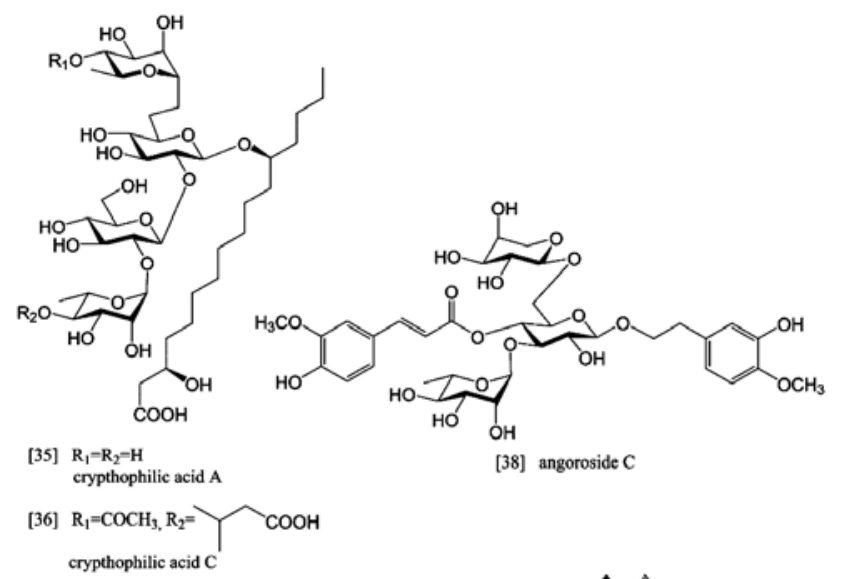

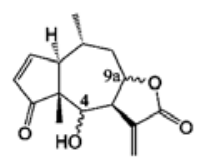

$\begin{array}{lll}{[43]} & 4 S, 9 a R & \text { helenalin } \\ {[44]} & 4 R, 9 a S & \text { mexicanin } \mathrm{I}\end{array}$

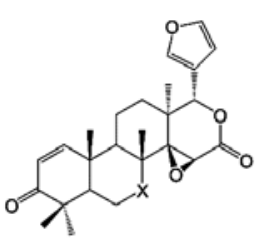

[48] $\mathrm{X}=\mathrm{CHOH} S$ [49] $\mathrm{X}$-deacetyldeguin 7-0x0-7-deacetyldeguin

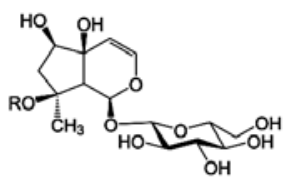

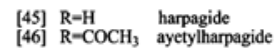

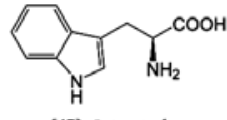

[47] L-tryptophan

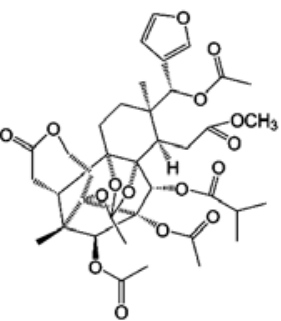

[50] kotschyin A

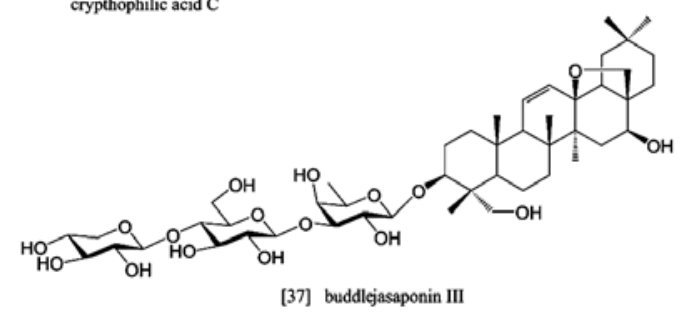

[37] buddlcjasaponin III

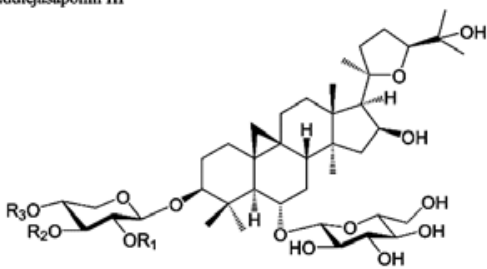

[39] $\mathrm{R}_{1}=\mathrm{R}_{2}-\mathrm{R}_{3}-\mathrm{COCH}_{3}$

[40] $R_{1}=R_{2}-R_{2}-C_{1} R_{3}=H$ stragloside I

[41] $\mathrm{R}_{1}-\mathrm{COCH}_{3} \mathrm{R}_{2}-\mathrm{R}_{3}=\mathrm{H}$ astragaloside II

$\begin{array}{ll}\text { [41] } \mathrm{R}_{1}-\mathrm{COCH}_{3}, \mathrm{R}_{2}-\mathrm{R}_{3}=\mathrm{H} & \text { astragaloside II } \\ \text { [42] } \mathrm{R}_{1}=\mathrm{R}_{2}=\mathrm{R}_{3}=\mathrm{H} & \text { astragaloside III }\end{array}$

Figure 3. Chemical structures of glycosides with trypanocidal activity.

dysentery and venereal disorders and also used topically to deal with wounds and abscesses. The $\mathrm{IC}_{50}$ values of both compounds were 0.045 and $0.73 \mu \mathrm{M}$, respectively, on bloodstream forms of T. $b$. rhodesiense and SI values of 86.7 and 20.3 were observed when compared to KB cells (human oral epidermal cell line). For the reference drug pentamidine, an $\mathrm{IC}_{50}$ value of $0.016 \mu \mathrm{g} / \mathrm{ml}(0.047 \mu \mathrm{M})$ was obtained (56).

Glycosides (Fig. 3): Resin glycosides, crypthophilic acid A [35] and crypthophilic acid C [36] as well as buddlejasaponin III [37], were isolated from Scrophularia cryptophila (Scrophulariaceae). The compounds exhibited growthinhibitory effect on bloodstream forms of $T . b$. rhodesiense with $\mathrm{IC}_{50}$ values of $46.9,14.1$, and $9.7 \mu \mathrm{g} / \mathrm{ml}(51.8,13.5$ and $10.8 \mu \mathrm{M})$, repsectively. For the reference drug, melarsoprol, an $\mathrm{IC}_{50}$ value of $0.0098 \mu \mathrm{g} / \mathrm{ml}(0.025 \mu \mathrm{M})$ was obtained. None of the isolates was toxic to L6 cells (57).

Angoroside C [38], a phenylethanoid glycoside, was isolated from the roots of Scrophularia lepidota (Scrophulariaceae), a plant endemic of the Turkish flora. The $\mathrm{IC}_{50}$ value was $29.3 \mu \mathrm{g} / \mathrm{ml}(36.3 \mu \mathrm{M})$ on bloodstream forms of T. b. rhodesiense. For the reference drug, melarsoprol, an $\mathrm{IC}_{50}$ value of $0.0033 \mu \mathrm{g} / \mathrm{ml}(0.008 \mu \mathrm{M})$ was obtained. No toxicity to L6 cells was observed (58).

Acetylastragaloside I [39] and astragalosides I, II, and IV $[40,41,42]$ were isolated from the roots of Astragalus

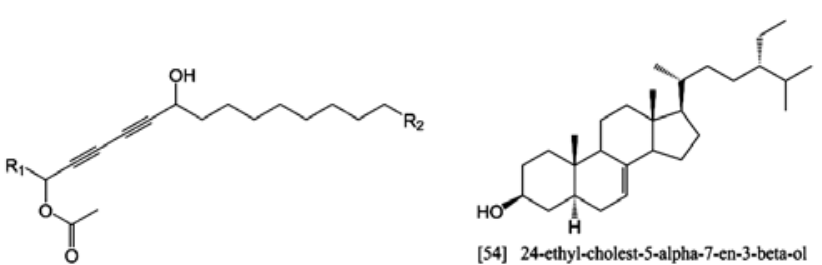

[51] $\mathrm{R}_{1}=\mathrm{CH}_{2} \mathrm{CH}_{3}, \mathrm{R}_{2}=\mathrm{CH}_{3}$

8-hydroxyheptadeca-4,6-diyn-3-yl acetate

[52] $\mathrm{R}_{1}-\mathrm{CHCH}_{2}, \mathrm{R}_{2}=\mathrm{CH}_{3}$

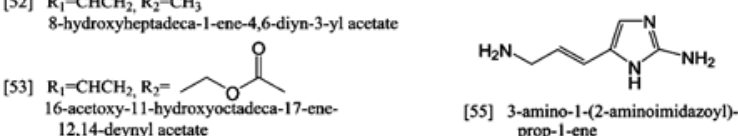
16-acetoxy-11-hydroxyoctadeca-17-ene-
12,14-deynyl acetate

[54] 24-ethyl-cholest-5-alpha-7-en-3-beta-ol

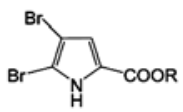

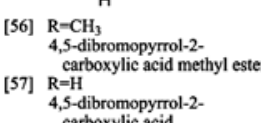
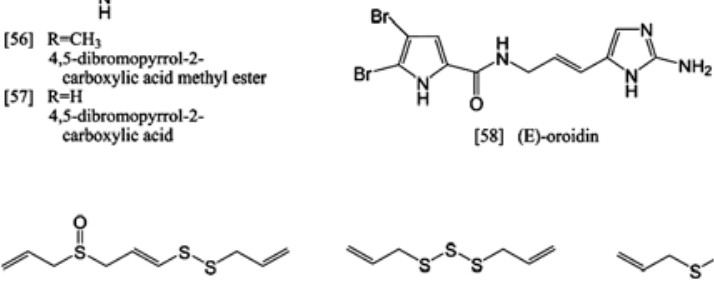

[59] ajoene

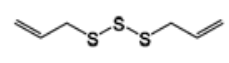

[60] diallyl trisulfide

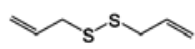

[61] diallyl disulfide

Figure 4. Chemical structures of terpenes and other metabolites with trypanocidal activity.

baibutensis (Leguminosae) and have been obtained earlier from A. trojanus. The $\mathrm{IC}_{50}$ values were 9.5, 57.6, 44.8 and $46.9 \mu \mathrm{g} / \mathrm{ml}(10.3,66.1,54.1$ and $59.8 \mu \mathrm{M})$, respectively, on bloodstream forms of $T . b$. rhodesiense. In the applied concentrations the most potent compound, acetylastragaloside I, possessed cytotoxicity to L6 cells with a SI value of 2.5. For the reference drug, melarsoprol, an $\mathrm{IC}_{50}$ value of $0.00295 \mu \mathrm{g} /$ $\mathrm{ml}(0.008 \mu \mathrm{M})$ was observed (59).

Terpenes (Fig. 4): Helenalin [43] and mexicanin I [44] are sesquiterpene lactones from Arnica and Inula species (Asteraceae). The $\mathrm{IC}_{50}$ values on bloodstream forms of $T . b$. rhodesiense were 0.051 and $0.318 \mu \mathrm{M}$, respectively, and SI values of 19.5 and 7.7 were obtained when compared to L6 cells. For the reference drug, melarsoprol, an $\mathrm{IC}_{50}$ value of $0.008 \mu \mathrm{M}$ was observed. It has been suggested that the activity of the compounds is due to the presence of an $\alpha, \beta$-unsaturated carbonyl group, which alkylates biological 
nucleophils. Moreover, the compounds display two alkylation centres, a cyclopentenone and the $\alpha$-methylene- $\gamma$-lactone moieties, mediating interactions with sulphhydryl groups of various enzymes. This bifunctionality is hypothesised to increase the specificity towards the parasite compared to monofunctional sesquiterpene lactones, which possessed higher cytotoxicity than trypanocidal activity. In addition, stereochemistry seems to play an important role, since mexicanin I, a diastereoisomer of helenalin, was six times less active. The mode of action is anticipated to involve the trypanothione metabolism to increase oxidative stress in the parasite (60).

Other metabolites (Fig. 4): Harpagide [45], acetylharpagide [46] and L-tryptophan [47] were isolated from Scrophularia cryptophila (Scrophulariaceae). The compounds exhibited growth-inhibitory effect on bloodstream forms of $T . b$. rhodesiense with $\mathrm{IC}_{50}$ values of $21.0,26.9$ and $4.1 \mu \mathrm{g} / \mathrm{ml}$ (59.7, 68.3 and $20 \mu \mathrm{M})$, respectively. For the reference drug, melarsoprol, an $\mathrm{IC}_{50}$ value of $0.0098 \mu \mathrm{g} / \mathrm{ml}(0.025 \mu \mathrm{M})$ was obtained. None of the isolates was toxic on L6 cells (57).

7-deacetylgedunin [48] and 7-oxo-7-deacetylgedunin [49], both limonoid deguins, and kotschyin A [50], a phragmalin-type limonoid orthoacetate, were isolated from Pseudocedrela kotschyi (Meliacae), a tree distributed from Senegal to Sudan. In the Sub-Saharan region $P$. kotschyi is commonly handled as a medical plant and in particular, in Nigeria it is used to treat sleeping sickness. The compounds possessed trypanocidal activity on bloodstream forms of $T . b$. rhodesiense with $\mathrm{IC}_{50}$ values of $3.45,3.40$ and $11.60 \mu \mathrm{g} / \mathrm{ml}$ (7.8, 7.7 and $14.5 \mu \mathrm{M})$, respectively. In the applied concentrations 7-deacetylgedunin exhibited cytotoxicity to murine macrophage RAW cells with an SI value of 3.9. The $\mathrm{IC}_{50}$ value for the reference drug, melarsoprol, was $0.004 \mu \mathrm{g} / \mathrm{ml}$ $(0.010 \mu \mathrm{M})(61)$.

Several polyacetylenes were isolated from Cussonia zimmermannii (Araliaceae), a tree growing in Kenya and Tanzania. 8-hydroxyheptadeca-4,6-diyn-3-yl acetate [51], 8hydroxyheptadeca-1-ene-4,6-diyn-3-yl acetate [52] and 16acetoxy-11-hydroxyoctadeca-17-ene-12,14-diynyl acetae [53] displayed activity on bloodstream forms of $T . b$. rhodesiense with $\mathrm{IC}_{50}$ values of $18,0.46$, and $1.1 \mu \mathrm{M}$, respectively, and SI values of 3, 26.1 and 52.7 when compared to L6 cells. For the reference drug, melarsoprol, an $\mathrm{IC}_{50}$ value of $0.0058 \mu \mathrm{M}$ was observed. In traditional medicine, a decoction of the root of $C$. zimmermannii is used to treat malaria (62).

24-ethyl-cholest-5 $\alpha$-7-en-3- $\beta$-ol [54], 3-amino-1-(2aminoimidazoyl)-prop-1-ene [55], 4,5-dibromopyrrole-2carboxylic acid methyl ester [56], 4,5-dibromopyrrole-2carboxylic acid [57] and (E)-oroidin [58] were isolated from Agelas oroides (Agelasidae), a Turkish marine sponge. The compounds as well as the TFA salts of the two latter substances possessed trypanocidal activity on bloodstream forms of $T . b$. rhodesiense with $\mathrm{IC}_{50}$ values of $14.2,2.4,13.3,20.9,25.2$, 17.3 and $12.2 \mu \mathrm{g} / \mu 1 \mathrm{l}(35.3,17.4,47.0,77.7,65.8,44.5$ and $24.3 \mu \mathrm{M})$, respectively. For the reference drug, melarsoprol, an $\mathrm{IC}_{50}$ value of $0.0027 \mu \mathrm{g} / \mu \mathrm{l}(0.007 \mu \mathrm{M})$ was observed. In the applied concentrations oroidin, oroidin TFA salt, and 3-amino-1-(2-aminoimidazoyl)-prop-1-ene possessed cytotoxicity to L6 cells with SI values of 5.1, 6.3 and 0.3 , respectively. Nevertheless, the potential of the most trypanocidal compound 3-amino-1-(2-aminoimidazoyl)-prop-1-ene is limited due to its high cytotoxicity. In this study, oroidin was identified as an uncompetitive inhibitor of Plasmodium falciparum FabI enzyme, which catalyzes the final step of the chain elongation in the fatty acid synthesis (63).

Allium sativum (Liliaceae), called garlic, is used in traditional medicine against cardiovascular diseases, cancer, and infections. A major sulphur component of the garlic bulb is allicin (diallyl thiosulfinate), which exhibits antibacterial properties. A degradation product, ajoene [59], is well known for its inhibitory effect on platelet aggregation and its cytotoxic, antifungal, antiviral, antitrypanosomal, and antimalarial activity. It was shown that ajoene inhibits the cholesterol synthesis and additionally its interference with the thiol homeostasis is suggested. Another degradation product is diallyl trisulfide, used in China for the treatment of bacterial, fungal and parasitic diseases. The $\mathrm{IC}_{50}$ value of diallyl trisulfide [60] was $0.8-5.5 \mu \mathrm{g} / \mathrm{ml}(10-15 \mu \mathrm{M})$ when tested on several subspecies of $T$. brucei bloodstream forms, including $T . b$. rhodesiense. In concentrations $\leq 140 \mu \mathrm{M}$, no cytotoxicity was observed on fibroblasts (64). The oily extract of the bulb of A. sativum administered i.p. $5.0 \mathrm{mg} / \mathrm{ml}$ per day suppressed trypanosomal infections in mice. The acetic acid/methanol fraction, which was suggested to be maintained by dially disulfide [61], retained the trypanocidal effect of the crude extract and cured infected mice in i.p. application of $120 \mathrm{mg} /$ $\mathrm{kg}$ per day within four days. The authors anticipate that the trypanocidal effect was by interference with the parasites' synthesis of membrane lipids (65).

\section{Conclusion}

After prolonged exposure to drugs, parasites can frequently develop resistance towards chemotherapeutics. Natural products are a promising source for screening new drugs. Many compounds have been demonstrated to possess high trypanocidal activity and moderate cytotoxicity values towards mammalian cells in vitro, which might open avenues for further investigation. In addition, it has been shown that by derivatization of potent substances, related compounds with improved trypanocidal effects can be developed. This demonstrates the usefulness of natural products as novel lead structures as a basis for further drug development. Unfortunately, investigations in animal models as well as studies focusing on the mode of action of natural products towards trypanosomes are still rare.

The understanding of the molecular mode of action is of great importance to advance the development of potential new drugs. For example, use of microarray studies to show regulation of genes which are affected by drug treatment and to elucidate pathways in which the drug acts. When a target molecule is known, site-directed mutagenesis or QSAR (quantitative structure-activity relationship)-analysis are facilitating methods to reveal structure-activity-relationships, by investigation of the molecular properties which account for the biological action of a drug.

Although, a number of drug candidates have been investigated, research has still not continued to pass the initial in vitro investigations to a point of clinical studies. In our 
opinion, there is an urgent need for natural product research for the treatment of trypanosomal infections.

\section{References}

1. Tagboto $\mathrm{S}$ and Townson $\mathrm{S}$ : Antiparasitic properties of medical plants and other naturally occurring products. Adv Parasitol 50: 199-295, 2001.

2. Kayser O and Kiderlen AF: Delivery strategies for antiparasitics. Expert Opin Investig Drugs 12: 197-207, 2003.

3. Kayser O, Kiderlen AF and Croft SL: Natural Products as antiparasitic drugs. Parasitol Res 90 (Suppl 2): 55-62, 2003.

4. Holzgrabe U and Bechthold A: Paradigmenwechsel in der Entwicklung antiinfektiver Chemotherapeutika. J Chemother 8: 69-78, 1999.

5. Barrett MP: The fall and rise of sleeping sickness. Lancet 353: 1113-1114, 1999.

6. Tanowitz HB, Wittner M, Werner C, Weiss LM, Kirchhoff LV and Bacchi C: Trypanosomes. In: Encyclopedia of Microbiology. Lederberg J (ed). Vol. 4 Q-Z, 2nd edition, Academic Press, London, pp725-741, 2000.

7. World Health Organisation: African trypanosomiasis (sleeping sickness). Fact sheet No. 259 WHO, 2006.

8. Centers of Disease Control and Prevention, CDC's Division of Parasitic Diseases: African trypanosomiasis. http://www.dpd. cdc.gov/dpdx/HTML/TrypanosomiasisAfrican.htm, January 14 th, 2008.

9. Ali V and Nozaki T: Current therapeutics, their problems, and sulfur-containing-amino-acid metabolism as a novel target against infections by 'amitochondriate' protozoan parasites. Clin Microbiol Rev 20: 164-187, 2007.

10. Shapiro TA and Englund PT: The structure and replication of kinetoplast DNA. Annu Rev Microbiol 49: 117-143, 1995.

11. Opperdoes FR and Michels PAM: Enzymes of carbohydrate metabolism as potential drug targets. Int J Parasitol 31: 482-490, 2001.

12. Penketh PG and Klein RA: Hydrogen peroxide metabolism in Trypanosoma brucei. Mol Biochem Parasitol 20: 111-121, 1986.

13. Fairlamb A, Blackburn P, Ulrich P, Chait B and Cerami A: Trypanothione: a novel bis (glutathionyl) spermidine cofactor for glutathione reductase in trypanosomatids. Science 22: 1485-1487, 1985.

14. Bacchi CJ and Yarlett N: Effects of antagonists of polyamine metabolism on African trypanosomes. Acta Trop 54: 225-236, 1993.

15. Mäser P, Lüscher A and Kaminsky R: Drug transport and drug resistance in African trypanosomes. Drug Resist Updat 6: 281-290, 2003.

16. Fish WR, Looker DL, Marr JJ and Berens RL: Purine metabolism in the bloodstream forms of Trypanosoma gambiense and Trypanosoma rhodesiense. Biochim Biophys Acta 719: 223-231, 1982.

17. Coppens I and Courtoy PJ: The adaptative mechanisms of Trypanosoma brucei for sterol homeostasis in its different lifecycle environments. Annu Rev Microbiol 54: 129-156, 2000.

18. Matovu E, Stewart ML, Geiser F, Brun R, Mäser P, Wallace LJ, Burchmore RJ, Enyaru JC, Barrett MP, Kaminsky R, Seebeck T and de Koning HP: The mechanisms of arsenical and diamidine uptake and resistance in Trypanosoma brucei. Eukaryot Cell 2: 1003-1008, 2003.

19. Delespaux V and de Koning HP: Drugs and drug resistance in African trypanosomiasis. Drug Resist Updat 10: 30-50, 2007.

20. Carter NS and Fairlamb AH: Arsenical-resistant trypanosomes lack an unusual adenosine transporter. Nature 361: 173-175, 1993.

21. de Koning HP and Jarvis SM: Adenosine transporters in bloodstream forms of Trypanosoma brucei brucei: substrate recognition motifs and affinity for trypanocidal drugs. Mol Pharmacol 56: 1162-1170, 1999.

22. Fox KR, Sansom CE and Stevens MF: Footprinting studies on the sequence-selective binding of pentamidine to DNA. FEBS Lett 266: 150-154, 1990.

23. Edwards KJ, Jenkins TC and Neidle S: Crystal structure of a pentamidine-oligonucleotide complex: implications for DNAbinding properties. Biochemistry 31: 7104-7109, 1992.

24. Bitonti AJ, Dumont JA and McCann PP: Characterization of Trypanosoma brucei brucei $S$-adenosylmethionine decarboxylase and its inhibition by Berenil, pentamidine and methylglyoxal bis (guanylhydrazone). Biochem J 237: 685-689, 1986.
25. Vansterkenburg ELM, Coppens I, Wilting J, Bos OJM, Fischer MJE, Janssen LHM and Opperdoes FR: The uptake of the trypanocidal drug Suramin in combination with low-density lipoproteins by Trypanosoma brucei and its possible mode of action. Acta Trop 54: 237-250, 1993.

26. Pépin J and Milord F: The treatment of human African trypanosomiasis. Adv Parasitol 33: 1-47, 1994.

27. Wang CC: Molecular mechanisms and therapeutic approaches to the treatment of African trypanosomiasis. Annu Rev Pharmacol Toxicol 35: 93-127, 1995.

28. Blum J, Nkunku S and Burri C: Clinical description of encephalopathic syndromes and risk factors for their occurrence and outcome during melarsoprol treatment of human African trypanosomiasis. Trop Med Int Health 6: 390-400, 2001.

29. Whittle HC and Pope HM: Febrile response to treatment in Gambian sleeping sickness. Ann Trop Med Parasitol 66: 7-14, 1974.

30. Fairlamb AH, Henderson GB and Cerami A: Trypanothione is the primary target for arsenical drugs against African trypanosomes. Proc Natl Acad Sci USA 86: 2607-2611, 1989.

31. Cunningham ML, Zvelebil MJ and Fairlamb AH: Mechanism of inhibition of trypanothione reductase and glutathione reductase by trivalent organic arsenicals. Eur J Biochem 221: 285-295, 1994.

32. Kuzoe FAS: Current situation of African trypanosoiasis. Acta Trop 54: 153-162, 1993.

33. Iten M, Matovu E, Brun R and Kaminsky R: Innate lack of susceptibility of Ugandan Trypanosoma brucei rhodesiense to $D L$-alpha-difluoromethylornithine (DFMO). Ann Trop Med Parasitol 46: 190-194, 1994.

34. Iten M, Mett H, Evans A, Enyaru JC, Brun R and Kaminsky R: Alterations in ornithine decarboxylase characteristics account for tolerance of Trypanosoma brucei rhodesiense to D,L-alphadifluoromethylornithine. Antimicrob Agents Chemother 41: 1922-1925, 1997.

35. Mamont PS, Duchesne MC, Grove J and Bey P: Antiproliferative properties of $D L$-alpha-difluoromethylornithine in cultured cells. A consequence of the irreversible inhibition of ornithine decarboxylase. Biochem Biophys Res Commun 81: 58-66, 1987.

36. Poulin R, Lu L, Ackermann B, Bey P and Pegg AE: Mechanism of the irreversible inactivation of mouse ornithine decarboxylase by alpha-difluoromethylornithine. Characterisation of sequences at the inhibitor and coenzyme binding sites. J Biol Chem 267: 150-158, 1992.

37. Bacchi CJ, Garofano J, Ciminelli M, Rattendi D, Goldberg B, McCann PP and Yarlett N: Resistance to $D L$-alphadifluoromethylornithine by clinical isolates of Trypanosoma brucei rhodesiense - role of $S$-adenosylmethionine. Biochem Pharmacol 46: 471-481, 1993.

38. Weber JT and Courvalin P: An empty quiver: antimicrobial drugs and resistance. Emerg Infect Dis 11: 791-793, 2005.

39. Mäser P, Sütterlin C, Kralli A and Kaminsky R: A nucleoside transporter from Trypanosoma brucei involved in drug resistance. Science 285: 242-244, 1999.

40. Shahi SK, Krauth-Siegel RL and Clayton CE: Overexpression of the putative thiol conjugate transporter TbMRPA causes melarsoprol resistance in Trypanosoma brucei. Mol Microbiol 43: 1129-1138, 2002.

41. Mäser P and Kaminsky R: Identification of three ABC transporter genes in Trypanosoma brucei spp. Parasitol Res 84: 106-111, 1998

42. Keppler D, Leier I and Jedlitschky G: Transport of glutathione conjugates and glucuronides by the multidrug resistance proteins MRP1 and MRP2. Biol Chem 378: 787-791, 1997.

43. Barrett MP, Mottram JC and Coombs GH: Recent advances in identifying and validating drug targets in trypanosomes and leishmanias. Trends Microbiol 7: 82-88, 1999.

44. Tasdemir D, Kaiser M, Brun R, Yardely V, Schmidt TJ, Tosun F and Rüedi P: Antitrypanosomal and antileishmanial activities of flavonoids and their analogues: in vitro, in vivo, structureactivity relationship, and quantitative structure-activity relationship studies. Antimicrob Agents Chemother 50: 1352-1364, 2006.

45. Hoet S, Opperdoes F, Brun R and Quetin-Leclercq J: Natural products active against African trypanosomes: a step towards new drugs. Nat Prod Rep 21: 353-364, 2004.

46. Bringmann G, Saeba W, Rückert M, Miesa J, Michela M, Mudogob V and Brunc R: Ancistrolikokine D, a 5,8'-coupled naphthylisoquinoline alkaloid, and related natural products from Ancistrocladus likoko. Phytochemistry 62: 631-636, 2003. 
47. Bringmann G, Dreyer M, Faber JH, Dalsgaard PW, Staerk D, Jaroszewski JW, Ndangalasi H, Mbago F, Brun R and Christensen SB: Ancistrotanzanine C and related 5,1'- and 7,3'coupled naphthylisoquinoline alkaloids from Ancistrocladus tanzaniensis. J Nat Prod 67: 743-748, 2004.

48. Bringmann G, Dreyer M, Kopff H, Rischer H, Wohlfarth M, Hadi HA, Brun B, Meimberg H and Heubl G: ent-Dioncophylleine $\mathrm{A}$ and related dehydrogenated naphthylisoquinoline alkaloids, the first Asian dioncophyllaceae-type alkaloids, from the 'new' plant species Ancistrocladus benomensis. J Nat Prod 68: 686-690, 2005.

49. Labraña J, Machocho AK, Kricsfalusy V, Brun R, Codina C, Viladomata F and Bastida J: Alkaloids from Narcissus angustifolius subsp. transcarpathicus (Amaryllidaceae). Phytochemistry 60: 847-852, 2002.

50. Wright AD, Goclik E, König GM and Kaminsky R: Lepadins D-F: antiplasmodial and antitrypanosomal decahydroquinoline derivatives from the tropical marine tunicate Didemnum $s p$. J Med Chem 45: 3067-3072, 2002.

51. Copp RB, Kayser O, Brun R and Kiderlen AF: Antiparasitic activity of marine pyridoacridone alkaloids related to the ascididemins. Planta Med 69: 527-531, 2003.

52. Mbwambo ZH, Apers S, Moshi MJ, Kapingu MC, Van Miert S, Claeys M, Brun R, Cos P, Pieters L and Vlietinck A: Anthranoid compounds with antiprotozoal activity from Vismia orientalis. Planta Med 70: 706-710, 2004.

53. Weniger B, Vonthron-Sénécheaun C, Kaiser M, Brun R and Anton R: Comparative antiplasmodial, leishmanicidal and antitrypanosomal activities of several biflavonoids. Phytomedicine 13: 176-180, 2006

54. Wright AD, Papendorf $\mathrm{O}$ and König GM: Ambigol $\mathrm{C}$ and 2,4dichlorobenzoic acid, natural products produced by the terrestrial cyanobacterium Fischerella ambigua. J Nat Prod 68: 459-461, 2005.

55. Gertsch J, Tobler RT, Brun R, Sticher O and Heilmann J: Antifungal, antiprotozoal, cytotoxic and piscicidal properties of Justicidin B and a new arylnaphthalide lignan from Phyllanthus piscatorum. Planta Med 69: 420-424, 2003.

56. Moideen VSK, Houghton PJ, Rock P, Croft SL and AboagyeNyame F: Activity of extracts and naphthoquinones from Kigelia pinnata against Trypanosoma brucei brucei and Trypanosoma brucei rhodesiense. Planta Med 65: 536-540, 1999.
57. Tasdemir D, Brun R, Franzblau SG, Sezgin Y and Calís I: Evaluation of antiprotozoal and antimycobacterial activities of the resin glycosides and the other metabolites of Scrophularia cryptophila. Phytomedicine 15: 209-215, 2008.

58. Tasdemir D, Güner ND, Perozzo R, Brun R, Dönmez AA, Calís I and Rüedi P: Anti-protozoal and plasmodial FabI enzyme inhibiting metabolites of Scrophularia lepidota roots. Phytochemistry 66: 355-362, 2005

59. Calís I, Koyunoglu S, Yesilada A, Brun R, Rüedi P and Tasdemir D: Antitrypanosomal cycloartane glycosides from Astragalus baibutensis. Chem Biodivers 3: 923-929, 2006.

60. Schmidt TJ, Brun R, Willuhn G and Khalid SA: Anti-trypanosomal activity of helenalin and some structurally related sesquiterpene lactones. Planta Med 68: 750-751, 2002.

61. Hay AE, Ioset JR, Ahua KM, Diallo D, Brun R and Hostettmann K: Limonoid orthoacetates and antiprotozoal compounds from the roots of Pseudocedrela kotschyi. J Nat Prod 70: 9-13, 2007.

62. Senn M, Gunzenhauser S, Brun R and Séquin U: Antiprotozoal polyacetylenes from the Tanzanian medicinal plant Cussonia zimmermannii. J Nat Prod 70: 1565-1569, 2007.

63. Tasdemir D, Topaloglu B, Perozzo R, Brun R, O'Neill R, Carballeira NM, Zhang X, Tonge PJ, Linden A and Rüedi P: Marine natural products from the Turkish sponge Agelas oroides that inhibit the enoyl reductases from Plasmodium falciparum, Mycobacterium tuberculosis and Escherichia coli. Bioorg Med Chem 15: 6834-6845, 2007.

64. Lun ZR, Burri C, Menzinger M and Kaminsky R: Antiparasitic activity of diallyl trisulfide (Dasuansu) on human and animal pathogenic protozoa (Trypanosoma sp., Entamoeba histolytica and Giardia lamblia) in vitro. Ann Soc Belg Med Trop 74: $51-59,1974$.

65. Nok AJ, Williams S and Onyenekwe PC: Allium sativuminduced death of African trypanosomes. Parasitol Res 82: 634-637, 1996. 